Uniciencia. Vol. 29, No. 1, pp. 42-57. Enero, 2015.

ISSN Electrónico: 2215-3470

URL: www.revistas.una.ac.cr/uniciencia

Email: revistauniciencia@una.cr

DOI: http://dx.doi.org/10.15359/ru.29-1.3

\title{
Aplicación de Actividades de modelización matemática en la educación secundaria costarricense
}

\author{
Application of Mathematical Modeling Activities in Costarican High School Education
}

Karen Porras-Lizano

krenporraslizano@yahoo.es

Escuela de Matemática

Universidad Nacional. Heredia, Costa Rica

Jennifer Fonseca-Castro

jennifer.fonseca.castro@una.cr

Escuela de Matemática

Universidad Nacional. Heredia, Costa Rica

Fecha de recepción del artículo: 23 de abril de 2014.

Fecha de revisión del artículo: 17 de julio de 2014.

Fecha de aprobación del artículo: 3 de agosto de 2014.

\section{Resumen}

Este artículo describe la experiencia obtenida al implementar actividades de modelización matemática como estrategia metodológica, en la enseñanza de los temas de proporciones y semejanza, con un grupo de octavo año de un centro educativo diurno académico, situado en el sector de Desamparados en la provincia de San José de Costa Rica, en 2012. Se aplicaron diversas técnicas para la recolección de la información tales como las observaciones participantes y cuestionarios. Entre los resultados relevantes están el desarrollo cíclico del pensamiento matemático de los estudiantes participantes en las etapas del proceso de modelización matemática (descripción, manipulación, predicción y validación) durante la solución de las situaciones problemas propuestas; el desarrollo de habilidades para trabajo en equipo; y la apreciación de la matemática como una disciplina útil y eficaz por parte del estudiantado. Al resolver las actividades propuestas en este estudio, se generaron interacciones sociales como el compartir información, pensamientos e ideas matemáticas, que estimularon la zona de desarrollo próximo de los estudiantes participantes. Igualmente, las actividades de modelización matemática permitieron a los estudiantes tener una conducta positiva en las clases de matemática, lo que estimuló, a su vez, una actitud diferente en comparación con las clases regulares.

Palabras claves: Modelización matemática; resolución de problemas; estrategia metodológica.

Karen Porras Lizano y Jennifer Fonseca Castro

Artículo protegido por licencia Creative Commons: BY-NC-ND / Protected by Creative Commons: BY-NC-ND

Revista de acceso y publicación gratuita/ Access and publication in Uniciencia is totally no fee. 


\begin{abstract}
This paper describes the experience gained in implementing mathematical modeling activities as a methodological strategy in teaching issues such as proportions, with a group of eighth year of an academic-day-school, located in the province of San Jose, Costa Rica in 2012. Different techniques for gathering information were applied, such as participant observation and questionnaires. Among the relevant results are the cyclical development of mathematical thinking of students in the stages of mathematical modeling (description, manipulation, prediction and validation) for solving the problem; developing of teamwork skills; and appreciation of mathematics as a useful and effective discipline. To resolve the activities proposed in this study, social interactions such as sharing information, thoughts and ideas, were generated, stimulating the zone of proximal development of the participating students. Likewise, the mathematical modeling activities allowed students to have a positive role in mathematics classes, stimulating, in turn, a different attitude compared to regular classes.
\end{abstract}

Keywords: mathematical modeling; problem solving; methodological strategy.

Ante una sociedad globalizada, la educación de las generaciones actuales debe asumir grandes retos competitivos, lo que demanda la exigencia de una excelente preparación académica. Para ello, la matemática es un pilar importante en la vida del ser humano. Esta disciplina fomenta habilidades y destrezas de gran significado en el desempeño del diario vivir, tales como el razonamiento lógico y la creatividad. Sin embargo, muchos estudiantes de secundaria cuestionan la utilidad de los temas del currículo de matemática y muestran su insatisfacción y desmotivación hacia esta.

Ante esta situación, se hace necesario introducir en el aula metodologías que ayuden al estudiante a apreciar la matemática como una disciplina útil y eficaz. Es decir, que experimenten una matemática más aplicable a su contexto, de manera que contribuya a disminuir la concepción errónea de ver la matemática desligada de la realidad circundante.

En esta línea, el Ministerio de Educación Pública de Costa Rica (MEP) pone en marcha en 2013 el nuevo Programa de Estudio en Matemática para la Educación General Básica y el Ciclo Diversificado (MEP, 2012), en el cual propone la resolución de problemas como estrategia pedagógica asociada a situaciones reales, donde la identificación, uso y construcción de modelos matemáticos sea parte sustancial y significativa en la labor de aula.

Desde esta perspectiva, el objetivo principal de la educación es que el estudiantado construya un aprendizaje con una comprensión profunda y rica de los conceptos matemáticos estudiados. La modelización matemática permite al personal docente considerar el entorno físico y social para abordar "situaciones problema" en contextos vinculados con los estudiantes, así como favorecer "la comprensión de conceptos, ya que los estudiantes percibirán a la matemática como una disciplina íntimamente relacionada con su cotidianidad" (Arguedas y Porras, 2008, p. vii), valorando así la importancia de los conceptos matemáticos enseñados.

Karen Porras Lizano y Jennifer Fonseca Castro

Artículo protegido por licencia Creative Commons: BY-NC-ND / Protected by Creative Commons: BY-NC-ND

Revista de acceso y publicación gratuita/ Access and publication in Uniciencia is totally no fee. 
El aprendizaje adquirido mediante la modelización contribuye a que el estudiantado visualice lo que aprende de una manera útil (Skemp, 1999), a la vez que aplica los conceptos cuando los necesita de manera flexible en nuevas situaciones. Esto proporciona una sensación gratificante para él, viéndose beneficiada su autoestima y el control del manejo de los conceptos involucrados.

El propósito de este artículo es presentar los resultados de una investigación llevada a cabo en 2013 en un colegio público diurno de la provincia de San José, donde se hace uso de la modelización matemática como estrategia metodológica en la enseñanza del tema de proporciones y semejanza. Para esto, se diseñaron e implementaron actividades de modelización matemática (AMM) obteniendo resultados interesantes en los ámbitos cognitivos, sociales y personales por parte del estudiantado.

\section{Marco teórico}

\section{Problemas tradicionales versus problemas de modelización matemática}

A lo largo de la vida del ser humano, es muy común enfrentarse a situaciones cotidianas que requieren de respuestas automáticas, cuyo proceso no es tan elaborado. Sin embargo, continuamente, se enfrentan situaciones que provocan una reacción diferente, no rutinaria y más elaborada. A esta última situación, es a lo que se le denomina un problema. Más específicamente, un problema es "una tarea que plantea a un individuo la necesidad de hallar una solución, sin contar con un procedimiento directamente accesible que la garantice" (Ortiz, 2001, p. 57).

Sin embargo, en el aula de matemática, resolver un problema tiende a confundirse con actividades como el solucionar ejercicios o problemas con contexto, que al final es un ejercicio rutinario.

En contraposición, algunos autores han utilizado la resolución de problemas como estrategia pedagógica para la enseñanza y aprendizaje de conceptos matemáticos (Schroeder y Lester, 1989). En este sentido, los problemas de modelización matemática juegan un papel primordial, creando la "situación-problema" que dará sentido al aprendizaje. Desde esta perspectiva, el "problema de modelización" o "situación-problema" debe permitir al estudiantado definir, refinar, transformar y extender su sistema conceptual para crear interpretaciones acertadas de la situación planteada. Este debe involucrar sistemas matemáticos lo más interesantes posibles, de manera que motiven al estudiante en la búsqueda y elaboración de modelos para entender y explicar la situación-problema (Lesh y Doerr, 2003).

El producto final es la unión de modelos complejos o herramientas conceptuales que revelan aspectos importantes de la forma de pensar del estudiante y que pueden ser reusados en situaciones similares (Lesh y Yoon, 2004).

El proceso involucrado en la resolución de la situación-problema debe contribuir en la búsqueda de soluciones, la exploración de patrones y la formulación de conjeturas, para dejar de lado la memorización de los procedimientos o fórmulas y la realización de ejercicios rutinarios (Grouws, 1992).

Karen Porras Lizano y Jennifer Fonseca Castro

Artículo protegido por licencia Creative Commons: BY-NC-ND / Protected by Creative Commons: BY-NC-ND

Revista de acceso y publicación gratuita/ Access and publication in Uniciencia is totally no fee. 
Ambos, el problema y el producto final, deben facilitar la reflexión del estudiantado mediante la documentación de su progreso y formas de pensar (Lesh, Hoover, Hole, Nelly y Post, 2000). Finalmente, la situación-problema debe proveer criterios para la evaluación alternativa de soluciones, así como para que el estudiante juzgue por sí mismo su producto final (Lesh et. al., 2000).

Así, la dinámica de la resolución de la situación-problema consiste en interpretar, buscar, seleccionar y aplicar herramientas conceptuales para entender y explicar la situación propuesta. Este es un proceso cíclico de expresión, interpretación, definición, transformación y extensión de ideas y conceptos, los cuales son gradualmente ordenados, integrados, refinados y procesados (Lesh y Yoon, 2004; Zawojewski y Lesh, 2003).

Es claro que la bibliografía e investigaciones han hecho su trabajo en definir y caracterizar el tipo de experiencias situaciones-problemas que pueden hacer la diferencia en el proceso de enseñanza-aprendizaje de la matemática. La dificultad está en encontrar, seleccionar o elaborar dichas situaciones-problemas.

En esa línea, recientes investigaciones han centrado sus esfuerzos en la elaboración de problemas de modelización que cumplan con las características antes descritas. Lesh y colegas, por ejemplo, trabajan en Estados Unidos de Norteamérica en la elaboración e implementación de actividades de modelización matemática (AMM) o MEAs (por sus siglas en Inglés, Model-Eliciting Activities). Dichas actividades han mostrado su potencial y alcance en la enseñanza y aprendizaje de las matemáticas en los distintos niveles escolares (Lesh y Doerr, 2003).

\section{Actividades de modelización matemática (AMM)}

Las AMM son actividades diseñadas en la línea de situación-problema descrita anteriormente. Estas le permiten al estudiantado definir, explorar y expresar sus ideas y conceptos en pequeños grupos de trabajo (de tres estudiantes), en aproximadamente 60 minutos. Estimulan en él la elaboración de modelos que permiten explicar un problema de la vida real, donde la información no siempre se presenta de forma explícita o en forma matematizada, y cuya solución no siempre se resume a aplicar un procedimiento o un algoritmo. Durante esos 60 minutos de trabajo, el estudiante hace uso de conocimientos previos, al mismo tiempo que los modifica y amplía (Lesh y Yoon, 2004).

Estas actividades cumplen con seis principios particulares que las diferencian de los problemas tradicionales: (1) el de significado personal, (2) el de construcción del modelo, (3) el de autoevaluación, (4) el de documentación, (5) el de prototipo simple, y (6) el de generalización del modelo. Estos principios aseguran que la situación-problema propuesta sea una situación del contexto del estudiantado, basada en situaciones de su vida real; de modo que genere, en él, la necesidad de construir, modificar, extender o refinar un modelo matemático como herramienta para resolver el problema propuesto. La actividad debe poseer criterios claros donde el estudiantado sea capaz de juzgar la utilidad y el progreso de las soluciones preliminares que surgen durante la resolución del problema; así como, especificaciones claras que le ayuden a documentar las construcciones que desarrolla; es decir, que permitan revelar explícitamente la forma en que está pensando acerca de la

Karen Porras Lizano y Jennifer Fonseca Castro

Artículo protegido por licencia Creative Commons: BY-NC-ND / Protected by Creative Commons: BY-NC-ND

Revista de acceso y publicación gratuita/ Access and publication in Uniciencia is totally no fee. 
situación propuesta. Debe motivar al estudiantado a formular o pensar en situaciones, en las que la solución propuesta del problema sirva, también, para resolver otros similares (Lesh y Doerr, 2003).

Las AMM vienen acompañadas de otras actividades de modelización que complementan el proceso. La figura 1 muestra dicha secuencia.

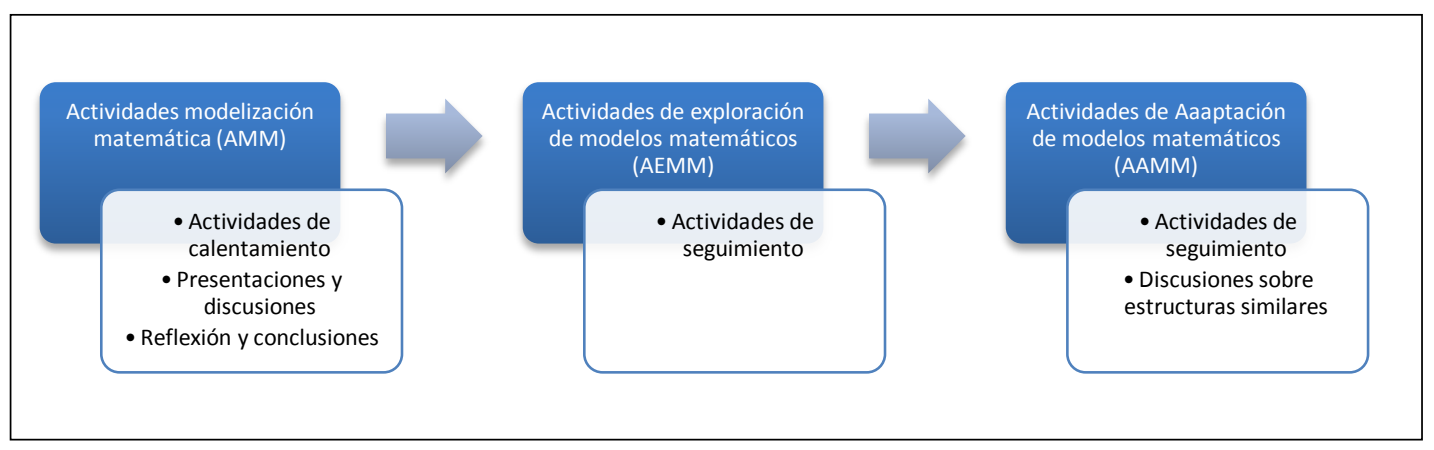

Figura 1.

Secuencia en el desarrollo de modelos matemáticos. Tomado de Lesh, Cramer, Doerr, Post, Zawojewski (2003).

Como muestra la figura anterior, las AMM están acompañadas de tres momentos: (1) La actividad de calentamiento, la cual se trabaja usualmente en la clase antes de trabajar la AMM y consiste en noticias de periódicos o revistas que ilustren y familiaricen al estudiantado con la situación-problema que trabajará en la AMM. (2) Presentaciones y discusiones, llevadas a cabo después de la sección de trabajo con la AMM. (3) Reflexión y conclusiones, donde el personal docente debe generar espacios de reflexión sobre lo que se logró hacer, debe institucionalizar las ideas y los conceptos matemáticos utilizados.

En las AEMM el objetivo es que el estudiantado desarrolle poderosos sistemas de representación y lenguaje para darle sentido al sistema conceptual propuesto, haciendo uso de herramientas tales como software, gráficos, diagramas, animaciones, entre otros.

Las AAMM, también conocidas como actividades de aplicación o extensión, tienen la finalidad de utilizar el modelo elaborado en la AMM y refinado en las AEMM, en situaciones similares a la presentada en la AMM, y que tal vez eran muy difíciles de resolver antes de la actividad de modelización.

Cabe destacar que muchas veces en el sector educativo, por diversos factores como tiempo, no se logra aplicar la secuencia de los tres tipos de actividades (AMM, AEMM, AAMM). Si bien el proceso completo es lo ideal, si se debe prescindir de alguna actividad, las últimas dos sirven de complemento y extensión de las AMM, por lo que el docente puede implementar las AMM y sustituir u omitir las otras dos actividades. 


\section{Marco metodológico Tipo de investigación y participantes}

La investigación objeto de este artículo se caracterizó por ser naturalista, debido a que se analizó el impacto de la modelización matemática en procesos de enseñanza y aprendizaje como alternativa metodológica en el aula de secundaria. Es decir, se estudió la forma en que los participantes abordan y dan importancia a las situaciones de aprendizaje cuando se utiliza la modelización matemática. El estudio se llevó a cabo en el primer trimestre del 2012.

Los participantes en este proceso fueron un grupo de 29 estudiantes cuya edad promedio es de 14 años; específicamente, una sección del nivel de octavo de un colegio académico diurno oficial, situado en la provincia en San José de Costa Rica en el cantón de Desamparados. Se escogió este nivel dado que los problemas de modelización diseñados abordan contenidos relacionados con proporciones y semejanza, temas propios de octavo año según el Programa de Matemática del Ministerio de Educación Pública (MEP, 2005). Parte de los criterios de la escogencia para la selección del grupo de estudiantes fueron: el horario de clases, las habilidades del grupo para trabajar en equipo, la iniciativa y los deseos de superación. Estos elementos contribuyen al desarrollo óptimo de destrezas importantes, tales como la creatividad y el dinamismo, muy importantes en este estudio, dada la temática marco.

También participó la docente-investigadora de matemática del grupo de estudiantes seleccionado; su colaboración resultó importante por su relación directa con el fenómeno de estudio y facilidad de ingreso al campo.

\section{Técnicas de recolección de información}

Se utilizaron la observación participante y el cuestionario como técnicas de recolección de información. En primer lugar, se aplicó la observación participante, esta se entendió como una técnica de recolección de datos interactiva, efectuada por la investigadora durante la implementación de las actividades de modelización matemática diseñadas para este estudio. El papel del investigador fue activo y participativo.

En segundo lugar, se aplicó un cuestionario de preguntas abiertas con el objetivo de obtener la percepción del grupo de estudiantes participantes acerca de la puesta en práctica de las actividades de modelización matemática. Además, esta técnica ayudó a corroborar los resultados obtenidos en las observaciones participantes.

\section{Instrumentos para la recolección de la información}

Parte fundamental en este proceso de investigación fueron los instrumentos que permitieron realizar el proceso de recolección de información en el trabajo de campo. Para el diseño de las actividades de modelización matemática, se consideraron aspectos como

Karen Porras Lizano y Jennifer Fonseca Castro

Artículo protegido por licencia Creative Commons: BY-NC-ND / Protected by Creative Commons: BY-NC-ND

Revista de acceso y publicación gratuita/ Access and publication in Uniciencia is totally no fee. 
los objetivos y contenidos concernientes a los temas de proporciones y semejanza de los Programas de Estudios del MEP de Tercer Ciclo (2005), dado que eran los programas que estaban en vigencia durante la investigación.

Las actividades utilizadas se adoptaron y algunas se rediseñaron a partir de las actividades de modelización matemática propuesta por Lesh et. al. (2000), en Principles for developing thought-revealing activities for students and teachers. Estas fueron modificadas acorde con los propósitos de este estudio, siempre buscando incorporar el contexto familiar a la vida del estudiantado. Dichas actividades se validaron mediante juicio de expertos y una prueba piloto con un grupo externo de estudiante del mismo nivel (ver anexo 1).

\section{Descripción del trabajo de campo}

El trabajo de campo se llevó a cabo en tres momentos. Cada una de las sesiones duró entre 2 y 4 lecciones de 40 minutos, este tiempo dependió del tipo de actividad que se estuviera desarrollando.

Un primer momento fue la implementación de la actividad de calentamiento, la canción "El costo de la vida". Se realizó una discusión en plenario con preguntas guías elaboradas por la docente-investigadora, cuyo objetivo principal era que el estudiantado brindara su opinión acerca del mensaje de la canción presentada.

En un segundo momento se le presentó al estudiantado la AMM "Calculando mi mesada". El estudiantado trabajó en la situación-problema en grupos de tres por aproximadamente tres lecciones de 40 minutos cada una. La asignación de los grupos de trabajo estuvo a cargo de la docente-investigadora quien conocía las cualidades de sus estudiantes. Cabe destacar que esta AMM fue la segunda experiencia de este tipo que los participantes enfrentaban, por lo que ya conocían la metodología de trabajo. Anteriormente, habían resuelto la actividad "Cocinando con mi mamá", cuyos resultados fueron presentados en el Octavo Congreso Internacional Sobre Educación Matemática Asistida por Computadora (CIEMAC), realizado en la provincia de Cartago, en Costa Rica, en 2013.

El tercer momento estuvo acompañado de las reflexiones y presentaciones. Se dio un espacio donde los grupos presentaron los informes o carteles a los demás compañeros. Se discutieron en forma general las soluciones de los grupos, evaluando en plenario la viabilidad de los métodos de solución. En esta misma etapa, se formalizaron los conceptos matemáticos involucrados en la actividad propuesta.

Cada sesión de trabaja fue video-grabada para facilitar el análisis posterior y para la triangulación de la información. Con esto, se buscó conocer las interacciones y otras conductas de los participantes durante la resolución de los problemas propuestos.

Después de la implementación de las actividades, se aplicó al grupo de estudiantes un cuestionario, para obtener información sobre la percepción acerca de la implementación de la modelización matemática como recurso metodológico.

Karen Porras Lizano y Jennifer Fonseca Castro

Artículo protegido por licencia Creative Commons: BY-NC-ND / Protected by Creative Commons: BY-NC-ND

Revista de acceso y publicación gratuita/ Access and publication in Uniciencia is totally no fee. 


\section{Resultados}

Dado que es la segunda vez que el grupo se enfrentaba a este tipo de actividades, los estudiantes se mostraron anuentes a colaborar, a descubrir, a generar ideas de resolución, a trabajar en equipo, como si estuvieran ante un reto por resolver. Las evidencias de lo anterior se describen a continuación:

\section{Momento 1: Actividad de calentamiento}

Se utilizó como actividad introductoria o de calentamiento la canción de Juan Luis Guerra "El costo de la vida". Esta creó mucha expectativa entre el estudiantado, quien por su experiencia anterior en la AMM “Cocinado con mi mamá" (Porras, 2013), sabían que la actividad se hacía con alguna intensión. Incluso algunos estudiantes manifestaron curiosidad, lo que se evidenció en preguntas como: “Profe que hizo el delantal?”, relacionando los contextos de ambas actividades mencionadas anteriormente.

Sus actitudes mostraban motivación, curiosidad e interés por el tema desarrollado en la canción.

Durante la actividad se les pidió a los estudiantes que brindaran su opinión acerca del mensaje de la canción presentada. La participación estudiantil fue enérgica, voluntaria y ordenada. Algunos comentarios fueron, "Todo crece, cada vez no alcanza la plata porque las cosas están más caras", "Si aumenta la gasolina también aumentan los pasajes de los autobuses", "Hay mucha corrupción pues los políticos se roban cada vez más la plata".

Además, se les preguntó sobre las consecuencias que puede ocasionar esta situación, a lo cual dieron respuestas como: "Despidos", "Falta de empleo", "Más delincuencia", "Protestas", entre otros.

\section{Momento 2: Resolución de la AMM}

Durante 60 minutos trabajaron los estudiantes y las estudiantes en grupos de tres en la solución de la actividad de modelización matemática, "Calculando mi mesada".

En relación con el desarrollo matemático de estudiantes, las observaciones permitieron evidenciar que iniciaban su análisis sacando la diferencia entre el precio actual de un artículo y el precio de hace diez años del mismo artículo. Erróneamente, nombraban esta diferencia como el aumento que tenían que hacerle a la mesada; sin embargo, esta hipótesis la rechazaban a los pocos minutos, pues una de las diferencias era bastante elevada. Uno de los estudiantes expresó: "Es ilógico que un papá aumentará tanto a la mesada del hijo”. Al rechazar la conjetura anterior, uno de los grupos ideó realizar una tabla con la cual compararon los precios de cada uno de los artículos y lograron observar que el precio actual, era aproximadamente tres veces el precio de hace diez años. Concluyeron así, que ese mismo comportamiento debía cumplirse al asignar el valor de la mesada. Es decir, que el monto actual debería ser tres veces la cantidad de dinero de la mesada de hace diez años (en clases posteriores, se utilizó este ejemplo para explicar la

Karen Porras Lizano y Jennifer Fonseca Castro

Artículo protegido por licencia Creative Commons: BY-NC-ND / Protected by Creative Commons: BY-NC-ND

Revista de acceso y publicación gratuita/ Access and publication in Uniciencia is totally no fee. 
Uniciencia. Vol. 29, No. 1, pp. 42-57. Enero, 2015.

URL: www.revistas.una.ac.cr/uniciencia

ISSN Electrónico: 2215-3470

Email: revistauniciencia@una.cr

DOI: http://dx.doi.org/10.15359/ru.29-1.3

razón de semejanza). De manera que corrigieron el error, logrando que la solución propuesta fuera perfeccionada y adecuada, al ser verificada en virtud de las condiciones del problema.

En el aspecto social, se observó que el estudiantado, al trabajar en equipo, compartía ideas y formas de pensar en relación con el problema. En ocasiones, no coincidían sus opiniones, pero con tolerancia y respeto lograron llegar a un consenso; por ejemplo, en uno de los grupos el estudiante "líder" formuló la conjetura de que "el precio actualmente del primer artículo era el triple del de hace 10 años", luego una compañera (Estudiante 1) pregunta, ¿Cómo sabes eso?”, él le explica, pero ella no logra entenderle. A raíz de esa situación se genera el siguiente diálogo:

Estudiante "lider": Todos estos artículos son de hace 10 años y todos estos son los mismos, pero actualmente (señala los artículos de la actividad).

Estudiante 1: Sí, sí eso sí lo entiendo.

Estudiante "líder": Son los mismos, los mismos. Le voy a explicar más despacio, antes, una mochila costaba casi 43000 colones. Ahora cuesta $\phi 9000$ colones.

Estudiante 2: Aquí multiplico (señalando los apuntes que Juan tenía en su cuaderno)

Estudiante 1: ¿Qué multiplicó?

Estudiante "líder": Yo multipliqué hasta que me diera el aproximado más cercano de este (señala el precio actual) que sería 3,4; más o menos, recuerda 3 por 3 es 9.

Estudiante "lider": El PlayStation valía 476000 colones, si lo multiplicamos por 3,3 (tomo una calculadora y le mostró el resultado a sus compañeros) entonces es $\phi 250$ 800 colones y el precio actual es $\phi 250750$ (tomo la hoja y señalo el precio actual), es exactamente el triple del otro precio de hace 10 años.

Estudiante 1: Ok, deme y yo hago el tercer artículo.

Estudiante 1: El precio de ahorita del combo es 3 veces el precio de hace 10 años.

Después del consenso en este grupo, se observó a la estudiante 1 , junto con sus otros dos compañeros, trabajando conjuntamente en su cuaderno, transcribiendo los resultados al informe que presentaron a los demás grupos.

Entre las dificultades que los grupos participantes tuvieron durante el proceso de resolución del problema estuvieron: la falta de tiempo, de comunicación (con compañeros que normalmente en las clases regulares no trabajan) y el ponerse de acuerdo en una solución para lograr éxito en la tarea planteada.

\section{Momento 3: Presentaciones}

En este espacio se realizaron las exposiciones de grupos. Cada grupo contó aproximadamente con 15 minutos para presentar su solución a los demás. La participación de los estudiantes en las presentaciones fue dinámica. Tanto los expositores como los

Karen Porras Lizano y Jennifer Fonseca Castro

Artículo protegido por licencia Creative Commons: BY-NC-ND / Protected by Creative Commons: BY-NC-ND

Revista de acceso y publicación gratuita/ Access and publication in Uniciencia is totally no fee. 
Uniciencia. Vol. 29, No. 1, pp. 42-57. Enero, 2015.

URL: www.revistas.una.ac.cr/uniciencia

ISSN Electrónico: 2215-3470

Email: revistauniciencia@una.cr

DOI: http://dx.doi.org/10.15359/ru.29-1.3

oyentes mostraron un cambio positivo en su actitud, mejoría en el orden y la disciplina de aula en comparación con las clases regulares. Incluso estudiantes que regularmente no son participativos en clase se mostraron más enérgicos, entusiasmados y colaborativos.

Este momento permitió espacios para compartir soluciones, pero principalmente para revisar y modificar ideas matemáticas. Esto se aprecia en el siguiente diálogo:

Estudiante 3: En el problema se trataba de saber cuánto se le tenía que dar a Mario para la mesada actualmente, él quería demostrar el porqué tenía que aumentársele la mesada y no que fuera igual a lo que le daban a la hermana hace 10 años.

Estudiante 3: Lo que hicimos fue dividir los precios actuales entre los de hace diez años y nos dio 3 por ciento.

Estudiante 3: Hace diez años el salveque costaba $ф 995$.

Estudiante 4: ф2995 (dijo Kevin corrigiendo a Lucía).

Estudiante 3: Sí, sí (haciendo caso a la observación de su compañero de grupo) ф2995 y actualmente vale 49500 , lo dividimos el precio actual es de 49500 entre el precio de hace diez años y nos dio que aumentó 3 por ciento.

Estudiante 3: Igual Playstation y no más que aquí (señalando la tercera fila de la tabla elaborada) la pizza nos dio 2,8\%, lo aproximamos a $3 \%$.

Estudiante 3: El monto de la mesada para Mario ahora debe ser de 46000 , porque de hace 10 años a ahora cada cosa ha subido un 3\% aproximadamente, lo que quiere decir que la mesada debe subir ese 3\% lo que daría 46000.

Estudiante 5: Esto también se puede ver en los precios de los celulares, pues no son los mismo actualmente que hace un tiempo atrás.

En el diálogo se observa que el grupo, en su explicación, utilizó el 3\% erróneamente, a lo que se refirieron en la explicación, es a multiplicar por 3. Este error fue observado por otro estudiante quien le preguntó a la profesora si era lo mismo 3 veces a un $3 \%$, a lo cual la docente respondió, “¿Cuál es la diferencia entre las dos cantidades?”. El estudiante contestó: “Creo que es porque el 3 es un entero y el 3\% no lo es”. Este resultado se explicó con más detalle por parte de la docente al finalizar las presentaciones de los estudiantes con la formalización de los conceptos.

Otro grupo participante expuso la siguiente solución, y destacó un abordaje diferente para solucionar el problema propuesto en la actividad:

Karen Porras Lizano y Jennifer Fonseca Castro

Artículo protegido por licencia Creative Commons: BY-NC-ND / Protected by Creative Commons: BY-NC-ND

Revista de acceso y publicación gratuita/ Access and publication in Uniciencia is totally no fee. 


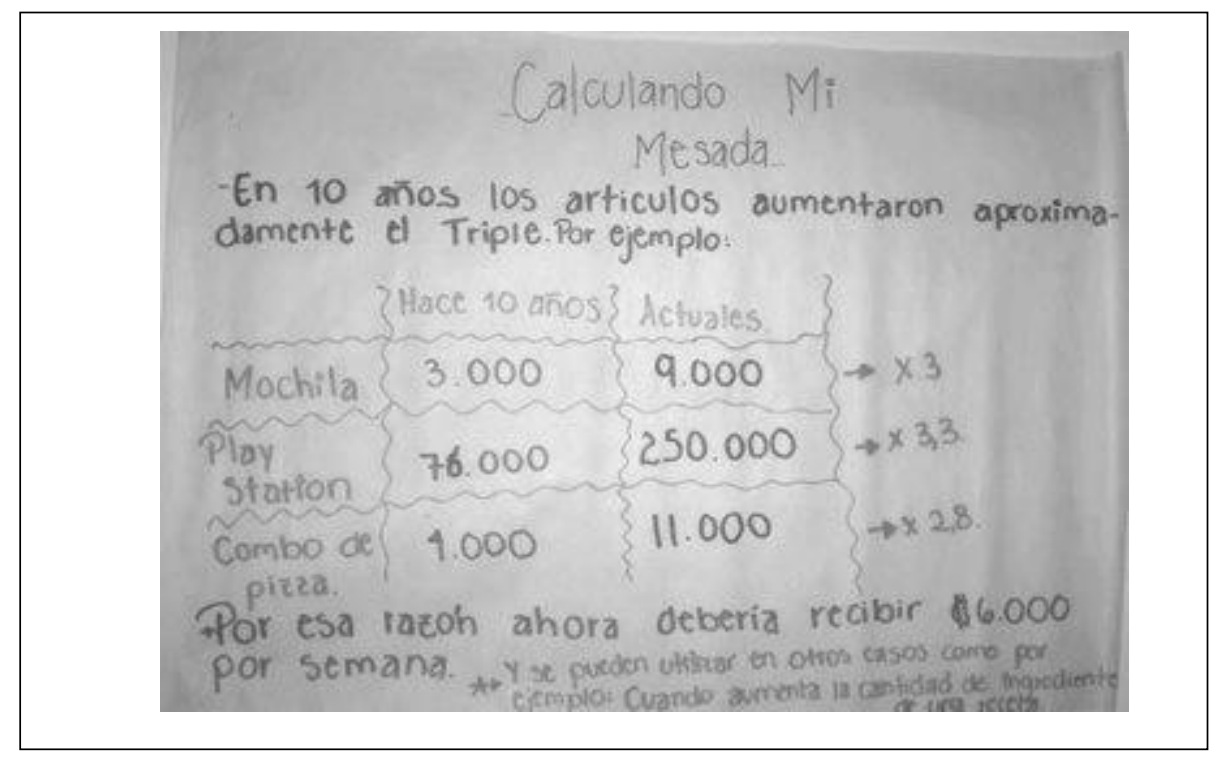

Figura 2.

La imagen muestra el informe de uno de los grupos, donde se observa el comportamiento de los precios de los artículos: una conjetura les permitió averiguar correctamente el monto aproximado de la mesada de Mario.

\section{Percepciones del estudiantado acerca de las actividades de modelización matemática}

Antes de terminar la puesta en práctica de las actividades formuladas para la investigación, se aplicó el cuestionario, donde se plantearon frases que el estudiante debía completar con su opinión en relación con las experiencias vividas en las clases anteriores.

El cuestionario reveló que el estudiantado visualiza claramente la utilidad de la matemática en situaciones cotidianas tales como para realizar cálculos de compras en lugares habituales de los pueblos (ejemplo la pulpería o supermercado), en los pagos de los recibos de electricidad, también para calcular la nota de un examen, entre otros.

De igual forma, destacaron la importancia de relacionar la matemática con otras materias como estudios sociales o ciencias. Incluso una estudiante expresó: " $L a$ matemática es indispensable para todo, se tiene que aplicar un poco de ella en todo". Además, mencionaron que al utilizar situaciones conocidas para enseñar los conceptos matemáticos, se sintieron mejor porque aprendieron más. En particular, un estudiante señaló, "Siento que es más fácil porque es algo que yo ya sé".

Asimismo, se les preguntó qué les parecía la matemática cuando se utiliza en problemas familiares para enseñarla, a lo cual, algunos estudiantes contestaron: "Es más 
Uniciencia. Vol. 29, No. 1, pp. 42-57. Enero, 2015.

URL: www.revistas.una.ac.cr/uniciencia

ISSN Electrónico: 2215-3470

Email: revistauniciencia@una.cr

DOI: http://dx.doi.org/10.15359/ru.29-1.3

fácil”, "sencilla, porque conozco el problema y logré resolverlo", "interesante, porque me intereso más en la materia”.

Para los estudiantes y las estudiantes, realizar actividades de este tipo, en grupo, les permitió según ellos mismos, pensar, dialogar e interactuar, experimentar múltiples sentimientos positivos. Además, mejoraron su estado emocional y su autoconcepto. Una estudiante expresó que al resolver el problema con elementos llamativos de su cotidianidad se sintió "capaz de hacer ese problema y muchos otros más”.

\section{Conclusiones}

Los resultados encontrados coinciden con la propuesta de Lesh y Doerr (2003), debido a que las actividades de modelización matemática permitieron al estudiantado desenvolverse en ciclos de definir, refinar, transformar y extender ideas y conceptos matemáticos con el propósito de entender y dar respuesta a la situación planteada.

La implementación de la AMM favoreció, en el estudiantado, el establecimiento de relaciones entre conceptos matemáticos, conocimientos previos y estrategias de resolución de problemas, así como conexiones con el entorno y aplicaciones de la vida cotidiana, como la construcción de situaciones ideadas por ellos mismos como parte de su realidad. Todo esto contribuyó, a que los estudiantes participantes en esta experiencia tuvieran la oportunidad de vivenciar las matemáticas de una forma diferente y significativa.

Aunado a lo anterior, se propició el trabajo en equipo en estudiantes, a través de un proceso social colaborativo, lo que les estimuló a la construcción de su conocimiento en forma activa, pues el abordaje de cada situación problema originó múltiples cuestionamientos y discrepancias de criterios entre participantes, enriqueciendo las discusiones de aula. Se destacó la importancia de la comunicación y el intercambio de ideas en la educación matemática, que estimularon lo que define Plaul (2012), haciendo alusión a la teoría de Vigotsky, como la zona de desarrollo próximo del estudiantado participante.

La AMM propuesta, generó un cambio de conducta positiva por parte de los grupos de estudiantes. Una actitud diferente en comparación con las clases regulares. Se observó interés y motivación hacia los conceptos desarrollados en cada actividad aplicada.

Acerca de la percepción estudiantil al utilizar la modelización matemática en el aula, se encontró que las situaciones cotidianas les permitieron visualizar la utilidad y facilidad del conocimiento matemático.

Es claro que poner en práctica metodologías como la que se propuso en este estudio implica un enorme compromiso por propiciar un desempeño positivo del educando en el aula. Desde esta perspectiva, el profesorado de matemática debe asumir el reto de innovarse constantemente, además de tener una buena disposición al enfrentarse a dificultades como el tiempo y comportamiento estudiantil.

Adicionalmente, con esta investigación, se buscó brindarles, a los estudiantes y las estudiantes, alternativas más atractivas que conduzcan a un aprendizaje que provenga del

Karen Porras Lizano y Jennifer Fonseca Castro

Artículo protegido por licencia Creative Commons: BY-NC-ND / Protected by Creative Commons: BY-NC-ND

Revista de acceso y publicación gratuita/ Access and publication in Uniciencia is totally no fee. 
Uniciencia. Vol. 29, No. 1, pp. 42-57. Enero, 2015.

URL: www.revistas.una.ac.cr/uniciencia

ISSN Electrónico: 2215-3470

Email: revistauniciencia@una.cr

DOI: http://dx.doi.org/10.15359/ru.29-1.3

interés del individuo, valorando la utilidad de la matemática a través de aplicaciones prácticas de los conceptos estudiados.

\section{Referencias}

Arguedas, A., y Porras, J. (2008). El uso del contexto sociocultural del estudiante como elemento facilitador de su aprendizaje sobre conceptos básicos de funciones en matemática (Tesis de licenciatura). Universidad Nacional, Heredia, Costa Rica.

Grouws, D. (Ed.). (1992). Handbook of research on mathematics teaching and learning. A Project of the national council of teachers of mathematics [Manual de investigación sobre la enseñanza de las matemáticas y el aprendizaje. Un proyecto del Consejo Nacional de Maestros de Matemáticas]. New York: Macmillian Publishing Company.

Lesh, R., Cramer, K., Doerr, H. M., Post, T. y Zawojewski, J. S. (2003). Model Development Sequences [Modelo de desarrollo de secuencias]. En R. Lesh y H. M. Doerr (Eds.), Beyond Constructivism: Models and Modeling Perspectives on Mathematics Problem Solving, Learning, and Teaching. Mahwash [Más allá del constructivismo: Perspectivas sobre Modelos y Modelado en la Solución de Problemas Matemáticos, Aprendizaje y Enseñanza.]. New Jersey: Lawrence Erlbaum Association.

Lesh, R., Hoover, M., Hole, B., Kelly, A. E., y Post, T. (2000). Principles for developing thought-revealing activities for students and teachers [Principios para el desarrollo de actividades de pensamiento para estudiantes y profesores] . En A. E. Kelly y R. Lesh (Eds.), Handbook of research design in mathematics and science education[Manual de diseño de la investigación en educación de la matemática y la ciencia], pp. 591-646. Mahwah, NJ: Lawrence Erlbaum Associates.

Lesh, R., y Doerr, H. M. (2003). Beyond Constructivism: Models and Modeling Perspectives on Mathematics Problem Solving, Learning, and Teaching[Más allá del constructivismo: Perspectivas sobre Modelos y Modelado en la Solución de Problemas Matemáticos, Aprendizaje y Enseñanza.]. Mahwash, New Jersey: Lawrence Erlbaum Association.

Lesh, R., y Yoon, C. (2004). What is distinctive in (Our Views about) Models \& Modeling Perspectives on Mathematics Problem Solving Learning and Teaching? [qué es lo distintivo en (desde nuestra perspectiva) en la Perspectiva de Modelos y Modelado sobre Solución de Problemas Matemáticos de Aprendizaje y Enseñanza?] En H. Henn, y W. Blue, (Eds.), ICMI Study 14: Applications and Modeling in Mathematics Education [Aplicaciones y Modelado en Educación Matemática], p. 151-160. Dortmund: Springer.

Karen Porras Lizano y Jennifer Fonseca Castro

Artículo protegido por licencia Creative Commons: BY-NC-ND / Protected by Creative Commons: BY-NC-ND

Revista de acceso y publicación gratuita/ Access and publication in Uniciencia is totally no fee. 
Uniciencia. Vol. 29, No. 1, pp. 42-57. Enero, 2015.

URL: www.revistas.una.ac.cr/uniciencia

ISSN Electrónico: 2215-3470

Email: revistauniciencia@una.cr

DOI: http://dx.doi.org/10.15359/ru.29-1.3

Ministerio de Educación Pública de Costa Rica (2012). Programas de estudio en matemática para la educación general básica y el ciclo diversificado. San José, Costa Rica: Autor

Ministerio de Educación Pública de Costa Rica. (2005). Programa de estudios matemática educación tercer ciclo. San José, Costa Rica: autor.

Ortiz, F. (2001). Los problemas y la construcción de aprendizaje, matemática estrategias de enseñanza y aprendizaje (pp. 56-65). México, D.F: Pax México.

Plaul, R. L. (14 de abril, 2012). Lev Vigotsky-Teoría socio-histórica [Mensaje en un blog]. Recuperado de http://redesoei.ning.com/profiles/blogs/lev-vigotsky-teor-asocio-hist-rica

Porras, K. (diciembre, 2013). Modelaje matemático como método de investigación en las clases matemáticas. Trabajo presentado en el Octavo Congreso Internacional de Enseñanza de la Matemática Asistida por Computadora (CIEMAC). Cartago, Costa Rica. Recuperado de http://www.cidse.itcr.ac.cr/ciemac/memorias/8CIEMAC/Ponencias/01_Porras_Kare n_Modelacion_Matematica.pdf

Schroeder, T. L., y Lester, F. K., Jr. (1989). Developing understanding in mathematics via problem solving[El desarrollo de la comprensión de las matemáticas a través de la resolución de problemas.] . In P. R. Trafton (Ed.), New directions for elementary school mathematics [Nuevas orientaciones de la matemática en primaria.] (p. 3142). Reston, VA: National Council of Teachers of Mathematics.

Skemp, R. (1999). Psicología del aprendizaje de las matemáticas. Madrid, España: Morata

Zawojewski, J. S., y Lesh, R. A. (2003). A Models and Modeling Perspective on Problem Solving. [Una perspectiva sobre modelos y modelados en resolución de problemas] En R. Lesh y H. M. Doerr (Eds.), Beyond Constructivism: Models and Modeling Perspectives on Mathematics Problem Solving, Learning, and Teaching [Más allá del constructivismo: Perspectivas sobre Modelos y Modelado en la Solución de Problemas Matemáticos, Aprendizaje y Enseñanza.]. Mahwash, New Jersey: Lawrence Erlbaum Association.

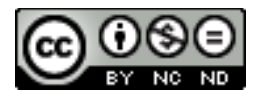

Aplicación de Actividades de Modelización Matemática en la Educación Secundaria Costarricense (Karen Porras Lizano y Jennifer Fonseca Castro) por Revista Uniciencia se encuentra bajo una Licencia CreativeCommons AtribuciónNoComercial-SinDerivadas 3.0 Unported.

Karen Porras Lizano y Jennifer Fonseca Castro

Artículo protegido por licencia Creative Commons: BY-NC-ND / Protected by Creative Commons: BY-NC-ND

Revista de acceso y publicación gratuita/ Access and publication in Uniciencia is totally no fee. 
Uniciencia. Vol. 29, No. 1, pp. 42-57. Enero, 2015.

URL: www.revistas.una.ac.cr/uniciencia

ISSN Electrónico: 2215-3470

Email: revistauniciencia@una.cr

DOI: http://dx.doi.org/10.15359/ru.29-1.3

\section{Anexo 1}

Actividad de modelización matemática: Calculando mi mesada. Tomado de Lesh, R.,

Hoover, M., Hole, B., Kelly, A. y Post, T. (2000)

Actividad de calentamiento: "El costo de la vida"

Como actividad introductoria o de calentamiento se les presentó a los estudiantes la canción de Juan Luis

Guerra "El costo de la vida", cuyo contexto es una crítica hacia la desigualdad económica que enfrentan muchos habitantes de América Latina; generándose problemas como la pobreza, diferencia social, exclusión, entre otros.

\section{Actividad de modelización matemática: "Calculando mi mesada"}

Se le presentó al estudiantado la situación-problema "Calculando mi mesada", cuyo propósito es que averigüen el aumento que se le debería hacer a la mesada de Mario (personaje de la actividad), quien recibe la misma cantidad de dinero que su hermana hace diez años. Mario cuestiona la cantidad que recibe, pues los artículos actualmente cuestan más que en esa época (ver precios abajo). El grupo de estudiantes debe justificar con argumentos válidos y lógicos la solución, pues debe convencer a los padres de Mario del aumento solicitado. La herramienta conceptual matemática involucrada en esta actividad es la proporcionalidad y comparación de medidas. Esta actividad cumple con los seis principios de modelización, pues, por ejemplo, la situación-problema tiene significado personal para el estudiantado, pues es una situación familiar para él; la misma presenta una necesidad, para él, de construir, modificar, extender o refinar su sistema conceptual como herramienta para resolver el problema propuesto. La actividad posee criterios claros para que el estudiantado juzgue la utilidad y el progreso de las soluciones preliminares, así como que documente las construcciones que desarrolla. Se desafío al estudiantado a ir más allá de una sola solución para que fuera capaz de producir otras maneras de pensar reutilizables, generalizando el modelo matemático de la situación problema.

iHola! Soy Mario, necesito tu ayuda con un problema acerca de mi mesada. Cuando mi
hermana Mariela tenía 13 años, recibía una mesada de $\phi 2000$, pero eso fue hace diez años.
Ahora yo tengo 13 años. Mis padres me dan $\phi 2000$ a la semana también. Creo que
deberían aumentar mi mesada teniendo en cuenta que las cosas cuestan más en la
actualidad. En Costa Rica con $\phi 2000$ ya no se puede comprar lo mismo que compraba mi
hermana hace diez años.
Para probar mi hipótesis he recogido los precios de hace 10 años de algunos artículos. De
igual forma, recolecté los precios de los artículos similares en la actualidad.
iEsto es lo que necesito de ustedes! Usen la información de los precios actuales y de hace

Karen Porras Lizano y Jennifer Fonseca Castro

Artículo protegido por licencia Creative Commons: BY-NC-ND / Protected by Creative Commons: BY-NC-ND

Revista de acceso y publicación gratuita/ Access and publication in Uniciencia is totally no fee. 
Uniciencia. Vol. 29, No. 1, pp. 42-57. Enero, 2015.

ISSN Electrónico: 2215-3470

URL: www.revistas.una.ac.cr/uniciencia

Email: revistauniciencia@una.cr

10 años para determinar, cuál debe ser el monto actual de mi mesada. Justifiquen su respuesta con razones lógicas pues mis padres no aceptarán argumentos emocionales o ilógicos.

Además expliquen el método de solución del problema para que sus compañeros en situaciones similares lo puedan utilizar para determinar cuáles deben ser sus entradas en la actualidad. Brinden 2 ejemplos de estas situaciones.

Precios hace 10 años
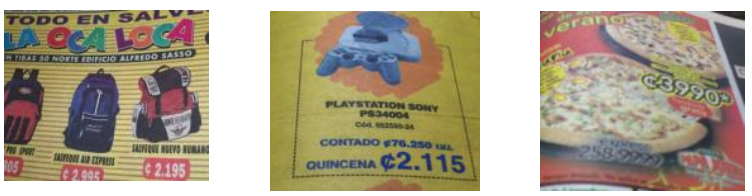

Precios después de 10 años
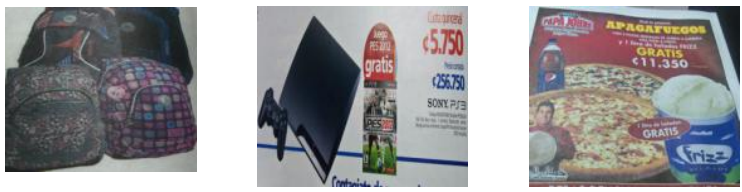